\title{
Analyzing bootsrap and foundation font-end frameworks: a comparative study
}

\author{
Majida Laaziri ${ }^{1}$, Khaoula Benmoussa ${ }^{2}$, Samira Khoulji ${ }^{3}$, Kerkeb Mohamed Larbi ${ }^{4}$, Abir EI Yamami ${ }^{5}$ \\ ${ }^{1,2,3}$ Information System Engineering Resarch Group, National School of Applied Sciences, \\ Abdelmalek Essaadi University, Morocco \\ ${ }^{4}$ Information System Engineering Resarch Group, Faculty of Sciences, Abdelmalek Essaadi University, Morocco \\ ${ }^{5}$ Laboratory Signals, Distributed Systems and Artificial Intelligence, ENSET Mohammedia,
} Hassan II University, Morocco

\section{Article Info \\ Article history: \\ Received Feb 18, 2018 \\ Revised Nov 20, 2018 \\ Accepted Dec 15, 2018}

\section{Keywords:}

Bootstrap

Foundation

Front-end frameworks

\begin{abstract}
Most modern web applications use some kind of front-end frameworks for designing and creating content in a faster and more efficient way, which saves valuable time when creating responsive web sites. There are many front-end frameworks that vary enormously in terms of features and benefits, which could make the choice of front-end framework for the developer tricky. In this context, this paper focuses on an effective analysis of two of today's most popular front-end frameworks, Boostrap and Foundation. The results show that our analysis can be beneficial for developers to select the appropriate front end framework to customize their web applications.
\end{abstract}

\section{Corresponding Author:}

Laaziri Majida,

Information System Engineering Resarch Group,

National School of Applied Sciences, Abdelmalek Essaâdi University,

Mhannech II, B.P 2121 Tetouan, Morocco.

Email: majida.laaziri@gmail.com

\section{INTRODUCTION}

Nowadays, web development is much simplified because of the emergence of different front-end frameworks. In recent years, dozens of frontend frameworks have become available to web developers. Front-end CSS development frameworks make the web development process faster, simpler and more standards-compliant. And help build a well-structured, maintainable and up-gradable website. Thus they also help the developer to save a lot of time because there is a multitude of already prebuilt elements ready to use. Each framework has its own strengths and weaknesses, and specific application areas, allowing the developer to choose one according to the needs of his specific project [1]. In addition, most of the options are modular, allowing to use only the components that the developer needs, or even mix components from different frameworks. There are so many framework choices but many developers are attracted to the most popular ones like Bootstrap and Foundation.

The authors [2] state in their study of Responsive Web Design Frameworks that front-end frameworks reduce the workload of developers and shorten a front-end development process. These frameworks help developers to quickly create a website. So they insist that the Bootstrap framework and Foundation have become the most remarkable frameworks by developers and most used. The author [3] in his article on Responsive Web Design with the Bootstrap Framework prefers to work with the bootsrap framework because it is a stylish, intuitive and powerful front end. Bootstrap has a standard set of classes that allow developers to quickly build applications. Bootstrap is the most popular html, css and javascript 
framework that offers flexibility in the use of tools without much knowledge of languages. Therefore, save time from coding heavy code. The author [4] confirms that Bootstrap uses LESS CSS, is compiled via Node, and is managed via GitHub to help developers make awesome things on the web. And Foundation is an advanced and responsive front-end framework. Foundation 3 is built with Sass, a powerful CSS preprocessor, which allows you to develop faster.

Studies have mounted that it has a debate on the choice of front end framework, in order to make the selection process easier, this article focuses on an effective analysis between the Bootstrap and Foundation frameworks which are the most used and popular. The reminder of this paper is presented as follow: section 2 presents a literature review about the subject, then we present trough section 3 our proposed model dedicated to the evaluation of web development frameworks. Section 4 presents a discussion of the obtained results, to sum up with a conclusion in section 5.

\section{LETARATURE REVIEW}

\subsection{Front-end framework}

The framework are common tools in the process of creation of web sites. There exist essentially two types of frameworks : front-end and back-end as shown in Figure 1, (this distinction is established according to whether the framework is for the presentation layer or the application/logical layer). The back-end of an application (a set of files with libraries to access databases, model structures, session management). There is many Back-end frameworks (i.e. Zend Framework, Symfony, Laravel, CakePHP ...)[5], [6].

A front-end framework is a library to ensure structure and graphical consistency very quickly. Structure in columns, typography (font, size, title, paragraph), components (button, form, dropdown, carousel, tab, tooltip, alert, modal), states, responsive ; they all bring a number of tools to save a lot of time in the creation of website [7]. Front-end frameworks typically consist of a package consisting of a standard code file and folder structure (HTML, CSS, JS documents, etc.).

The HTML language structures the data, the CSS stylizes this data to make them visually comprehensible and aesthetic, the JS (Javascript acronym) allows to produce animations and interactions improving the user experience. The usual components are [4], [8] :

a. CSS source code to create a grid: this allows the developer to position the different elements that make up the design of the site in a simple and versatile way.

b. Typography style definitions for HTML elements.

c. Solutions for browser incompatibility cases so that the site displays correctly in all browsers.

d. Create standard CSS classes that can be used to stylize advanced components of the user interface.

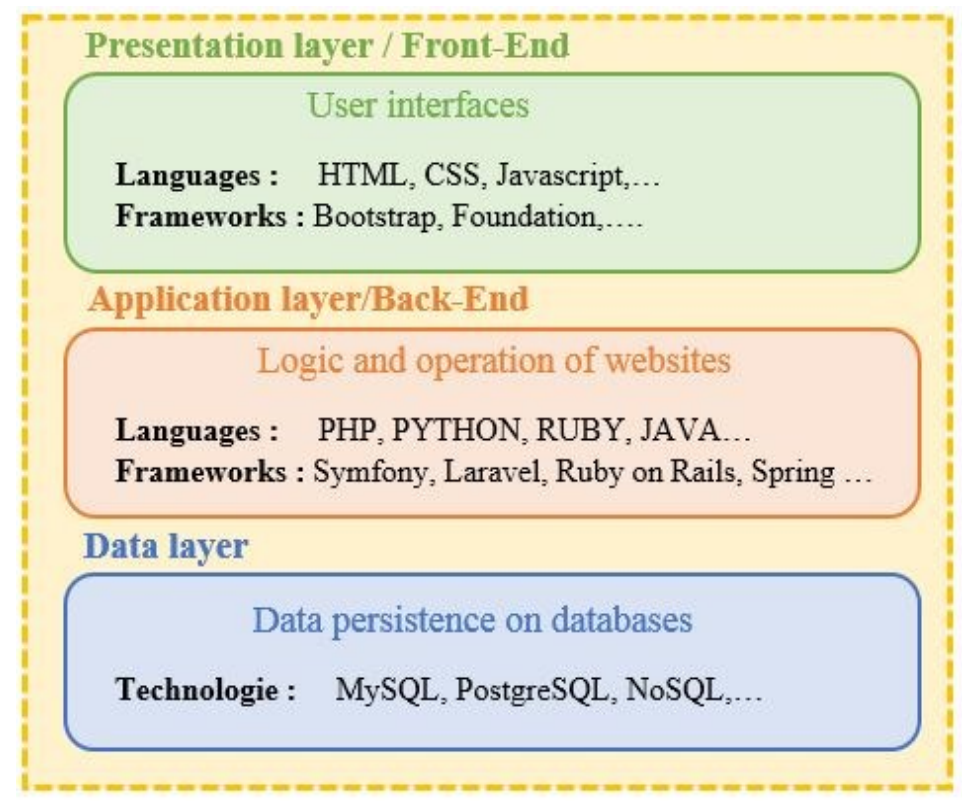

Figure 1. Back-end and front-end 
A front-end framework offers preformatted and configurable elements for the components of a web page:

a. A complete and manipulable layout

b. Modules covering all aspects and current needs

c. Standard states

d. Multiple interactive behaviors

e. A customizable responsive design

1. Time saving

We present blow the advantages of using a front-end framework derived from [5], [9] studies:

In the case of realization of a new project, the front-end developer must evaluate the development time but often the time presses. So thanks to the front-end framework the developer will not have to start from scratch. Rather than encode and create all the components of the page, it will have the task of assembling the preformatted elements and proposed by the framework. It's like eliminating a step in the front-end developer workflow.

2. Standardization and performance

A site developed using a framework will be efficient because the framework is optimized for the multiple web browsers of the market.

3. Scalability and update

The framework is regularly updated to comply with the latest web standards but also to offer relevant modules and up-to-date. Using a framework, we benefit from a set of beneficial improvements for the construction and implementation of a website that must comply with the latest standards.

4. Free and Open

Front-end frameworks are (at least currently) free and open. This means that they can be used without paying a license and being able to modify them at will. This is at least the case for Bootstrap and Foundation.

5. Reliability

The most popular frameworks by hundreds of thousands of developers who attest to the quality and reliability of the tool. The bugs are almost non-existent. In short, using a framework eliminates a sometimes laborious step that is to design the visual bases of the site and create different modules of zero. In addition to saving time, it provides a proven, robust, and scalable structure tested by thousands of users.

\subsection{Bootsrap}

Bootstrap Twitter is one of the best known and most used CSS frameworks as shown in Figure 2. It was developed on Twitter in 2010. This framework runs on a grid system of 12 columns, with 4 types of displays possible depending on the width of the user's screen. We can adapt the appearance of the site to the visitor's material. The Bootstrap offers components (buttons, navigation bars, form, ...) pretty and easy to integrate. Since version 3 , it is totally responsive. The elements adapt to the width of the screen by sliding one below the other when the width decreases. The adaptation is also done by a different display on the screen as we have already said. Its pre-programmed Queries media are now indispensable for smartphones. The bootstrap site offers many examples of directly usable templates. Bootstrap is associated with the Less preprocessor, a language that offers many features, such as using variables and including portions of code. It is thus possible to customize its CSS file with the elements and the colors wanted. The bootstrap of twitter is a safe bet in terms of CSS frameworks[10],[11]. Bootstrap offers :

a. a responsive grid, CSS styles for all standard HTML elements (form, button, text, image ...)

b. JS components such as dynamic drop-down lists, interactive navigation or carousel

c. a font that is actually a set of icons. The advantage is that the icons are not images but fonts, they are vectorized and therefore always sharp on all types of devices [3].

\subsection{Foundation}

Foundation was created by ZURB in 2008. It is a front end framework that consists of many useful tools for creating responsive and mobile first mobile sites. Primarily built with HTML, CSS, and jquery, Foundation uses modern techniques and practices, but degrades dramatically from Internet Explorer 8. Although the Framework is most commonly used by HTML and CSS developers, the developer has the opportunity to take its marks thanks to Foundation in collaboration with Sass and Rails. The company behind Foundation, ZURB, has created a rigorously regulated set of tools for developers. Each module has its role to play in the whole framework, but at the same time can function completely independently of the central leadership. What this means then is that the developer can combine and combine the features of his current project, or take a single feature and add it to an older project. It can even add it as a feature to a different Framework. In summary, this is a modern framework that has been well built and responds to a wide variety 
of users. It offers a good set of features. It's free, and there's a growing community of people to help the developers if they're stuck[10].

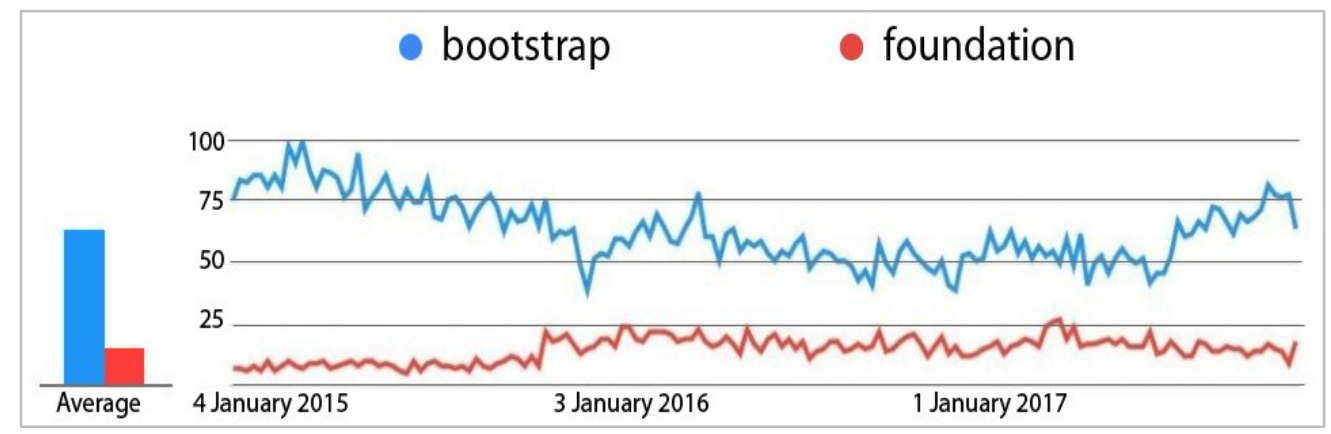

Figure 2. Statistic of the bootstrap and foundation front-end framework

\section{COMPARATIVE STUDY: BOOTSTRAP VS FOUNDATION}

We present below the similarities between the two frameworks, the results are based on [8], [5] studies.

a. Open source : Both frameworks work under MIT license, which means they are free to use and adapt.

b. Reactivity : Bootstrap and Foundation are reactive right out of the box. They apply the "mobile first" approach, which is a design methodology that indicates that it is best to first create the layout for smaller screens, then add more content and features as you go. as the screen grows.

c. 12-column grid system : In terms of frameworks, a grid system gives the developer the power to arrange their website or application as shown in Figure 3 and 4.

Both frameworks have :

a. Lines and columns

b. Ability to nest columns in rows, and vice versa

\begin{tabular}{|c|c|c|c|c|c|c|c|c|c|c|c|}
\hline $\begin{array}{l}\text {.col- } \\
\text { md-1 }\end{array}$ & $\begin{array}{l}\text {.col- } \\
\text { md-1 }\end{array}$ & $\begin{array}{l}\text { col- } \\
\text { md-1 }\end{array}$ & $\begin{array}{l}\text {.col- } \\
\text { md-1 }\end{array}$ & $\begin{array}{l}\text {.col- } \\
\text { md-1 }\end{array}$ & $\begin{array}{l}\text {.col- } \\
\text { md-1 }\end{array}$ & \begin{tabular}{|l} 
col- \\
md-1
\end{tabular} & \begin{tabular}{|l|}
.col- \\
md-1
\end{tabular} & $\begin{array}{l}\text {.col- } \\
\text { md-1 }\end{array}$ & $\begin{array}{l}\text {.col- } \\
\text { md-1 }\end{array}$ & $\begin{array}{l}\text {.col- } \\
\text { md-1 }\end{array}$ & $\begin{array}{l}\text {.col- } \\
\text { md-1 }\end{array}$ \\
\hline \multicolumn{6}{|c|}{.col-md-8 } & \multicolumn{3}{|c|}{.col-md-4 } & & & \\
\hline \multicolumn{4}{|c|}{.col-md-4 } & \multicolumn{4}{|c|}{.col-md-4 } & \multicolumn{4}{|c|}{.col-md-4 } \\
\hline \multicolumn{6}{|c|}{.col-md-6 } & \multicolumn{6}{|c|}{.col-md-6 } \\
\hline
\end{tabular}

Figure 3. Bootstrap grid example

\begin{tabular}{|c|c|c|c|c|c|c|}
\hline \multicolumn{7}{|c|}{.large-12.columns } \\
\hline \multicolumn{3}{|c|}{.large-6.columns } & \multicolumn{4}{|c|}{.large-6.columns } \\
\hline \multicolumn{2}{|c|}{.large-4.columns } & \multicolumn{2}{|c|}{ large-4.columns } & \multicolumn{3}{|c|}{.large-4.columns } \\
\hline \multicolumn{2}{|c|}{. large-3.columns } & large-3.columns & \multicolumn{2}{|c|}{ large-3.columns } & \multicolumn{2}{|c|}{. large-3.columns } \\
\hline $\begin{array}{l}\text { large- } \\
\text { 2.columns }\end{array}$ & $\begin{array}{l}\text {.. large- } \\
\text { 2.columns }\end{array}$ & $\begin{array}{l}\text { large- } \\
\text { 2.columns }\end{array}$ & $\begin{array}{l}\text { large- } \\
\text { 2.columns }\end{array}$ & & & $\begin{array}{l}\text {.. large- } \\
\text { 2.columns }\end{array}$ \\
\hline
\end{tabular}

Figure 4. Foundation grid example 
d. Stylized CSS components : Bootstrap and Foundation include additional CSS styles for different components of the web page, such as typography, tables, forms, navigation bar, buttons, pagination, labels, progress bar etc as shown in Figure. 5.

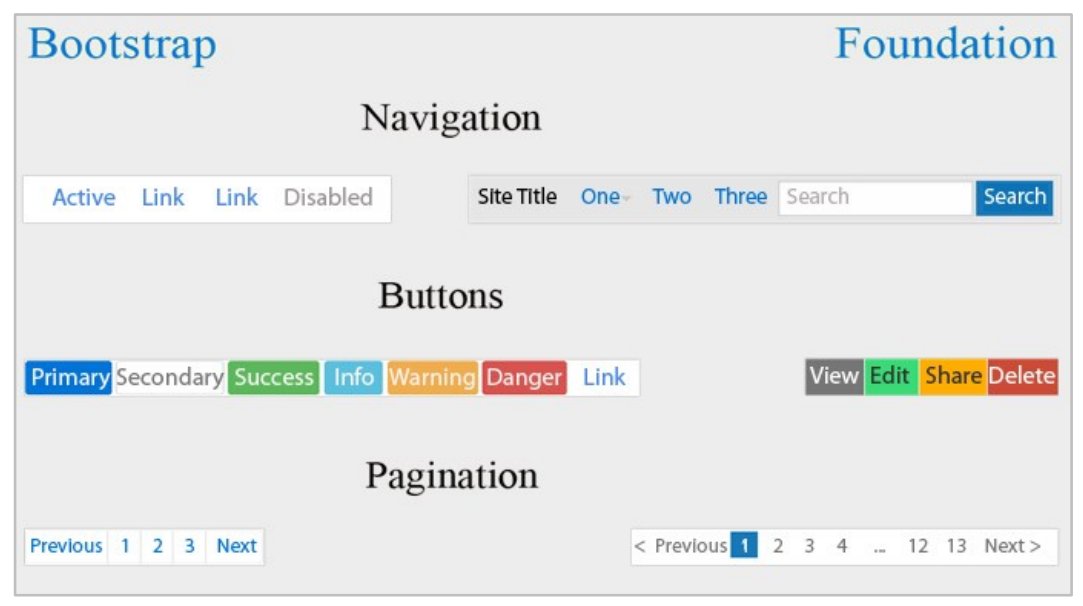

Figure 5. The different components of bootstrap and foundation

Table 1 present the basic characteristics of each Framework, a detailed description of each framework can be found in [12], [2]. Table 2 presents the CSS charateristics for each framework. Table 3 presents the charateristics related to grids and the responsiveness of each framework. Table 4 presents the charateristics related to the user interface (UI) and widgets of each framework.

Table 1. Bootstrap and Foundation Comparison

\begin{tabular}{ll}
\hline & \multicolumn{1}{c}{ Bootstrap } \\
\hline Owned by & Originally developed at Twitter by Mark Otto and Jacob \\
& Thornton, now an open-source project \\
Standard distribution & Required CSS (1 minified, 1 standard, both \\
package includes & with .map files); required JavaScript (1 minified, 1 \\
& standard). Former theme is incorporated in Sass files, \\
& activated by variables. (Glyphicons are not distributed \\
& with Bootstrap 4.) Also contains two additional sets of \\
& CSS files (1 minified, 1 standard, both with .map files): \\
& bootstrap-grid, which appears to be a flexbox-based grid \\
& system, possibly redundant at this point; and bootstrap- \\
& reboot, based on normalize.css. A CDN is available for \\
& standard distribution files.
\end{tabular}

Additional JavaScript libraries required?

Browser support

Internet Explorer support

Other browser support notes

License and copyright
Yes, you must download or link to a CDN separately: jQuery 3.1.1 Slim, Tether 1.4.0. Files are linked to just before end of body element.

Latest versions of: Chrome (macOS, Windows, iOS and Android), Safari (macOS and iOS), Firefox (macOS, Windows, Android, iOS) (latest version of Firefox plus Extended Support Release), Edge (Windows, Windows 10 Mobile), Opera (macOS, Windows), Android Browse \& WebView 5.0+, Windows 10 Mobile, Internet Explorer 10+

Internet Explorer 10 and higher (Bootstrap 3 recommended for Internet Explorer 8 and 9)

"Unofficially, Bootstrap should look and behave well enough in Chromium and Chrome for Linux, Firefox for Linux, and Internet Explorer 9, though they are not officially supported."

Code and documentation copyright (2011 to 2017) of the Bootstrap authors and Twitter, Inc. Code released under the MIT License. Docs released under Creative Commons.

Foundation
ZURB, a web development company in
Campbell, California

Four distributions : Complete, Essential,

Custom, Sass. Complete version includes compiled CSS (minified and not), plus compiled JavaScript, including individual vendor files. Essential includes typography, grid, Reveal, and Interchange only. Custom can be customized on the website for downloading, and the developer can choose elements to include and change a few variables. The Sass version can only be downloaded using the command line, Foundation CLI or Yeti Launcher. A CDN is available for standard distribution files.

All dependencies are bundled in the distribution. jQuery is required, but it is part of the distribution. Files are linked to just before end of body element.

Last two versions of Chrome, Firefox, Safari, Opera, mobile Safari, Internet Explorer mobile, as well as Internet Explorer 9+ and Android browser $2.3+$

\section{Internet Explorer 9 and higher}

"JavaScript: Our plugins use a number of handy ECMAScript 5 features that aren't supported in IE8."

MIT license, no mention of copyright 
Table 1. Bootstrap and Foundation Comparison

\begin{tabular}{|c|c|c|}
\hline & Bootstrap & Foundation \\
\hline
\end{tabular}

Table 2. CSS Comparison

\begin{tabular}{ccc}
\hline & Bootstrap & Foundation \\
\hline Reset & reboot.css(_reboot.scss) & normalize.css \\
LESS & Yes & No \\
Sass $/$ Scss & Yes & Yes \\
\hline
\end{tabular}

Table 3. Grids and Responsiveness Comparison

\begin{tabular}{|c|c|c|}
\hline & Bootstrap & Foundation \\
\hline \multirow{7}{*}{$\begin{array}{l}\text { Base width } \\
\text { Columns } \\
\text { Single column class syntax }\end{array}$} & Fluid $(0,34 \mathrm{em}, 48 \mathrm{em}, 62 \mathrm{em}, 75 \mathrm{em})$ & Fluid (max-width 75rem default) \\
\hline & 12 & 1-infinity (12 default) \\
\hline & $. \operatorname{col}-x s-1$ & .small-1.columns \\
\hline &. col-sm-1 & .medium-1.columns \\
\hline & .col-md-1 & . large-1.columns \\
\hline & $. \operatorname{col}-\lg -1$ & . [custom] -1. columns \\
\hline & $. \operatorname{col}-x 1-1$ & \\
\hline Container syntax & <div class="container"> & $<$ div class="row" > \\
\hline & or & \\
\hline & <div class="container-fluid"> & \\
\hline Nested column syntax & <div class="row" > & <div class="row" $>$ \\
\hline & $<$ div class="col-sm-9" > & <div class="small- 6 columns" > \\
\hline & $<$ div class="row" $>$ & <div class="row"> \\
\hline & $<!--$ nested columns add up & $<!--$ nested columns add up \\
\hline & to $12-->$ & to $12-->$ \\
\hline & $<$ div class=" col-xs-8 col- & $<$ div class $="$ small -6 \\
\hline & sm-6" $>\ldots</$ div $>$ & columns" $>\ldots</$ div $>$ \\
\hline & $<$ div class="col-xs-4 col- & $<\operatorname{div}$ class $="$ small -6 \\
\hline & sm-6" $>\ldots</$ div $>$ & columns" $>\ldots</$ div $>$ \\
\hline & $</$ div $>$ & $</$ div $>$ \\
\hline & $</$ div $>$ & $</$ div $>$ \\
\hline & $</$ div $>$ & $</$ div $>$ \\
\hline Visibility class syntax & Viewport-based: & Screen Size: \\
\hline & .hidden-xs-down & .show-for-medium \\
\hline & .hidden-sm-down & . show-for- [custom] \\
\hline & .hidden-md-down & .show-for-medium-only \\
\hline & .hidden-lg-down & . show-for-[ custom]-only \\
\hline & .hidden-xl-down & .hide-for-medium \\
\hline & .hidden-xs-up & .hide-for-[custom] \\
\hline & .hidden-sm-up & .hide-for-medium-only \\
\hline & .hidden-md-up & .hide-for-[custom] -only \\
\hline & .hidden-lg-up & \\
\hline & .hidden-xl-up & Generic (CAUTION: hides from \\
\hline
\end{tabular}


Table 3. Grids and Responsiveness Comparison

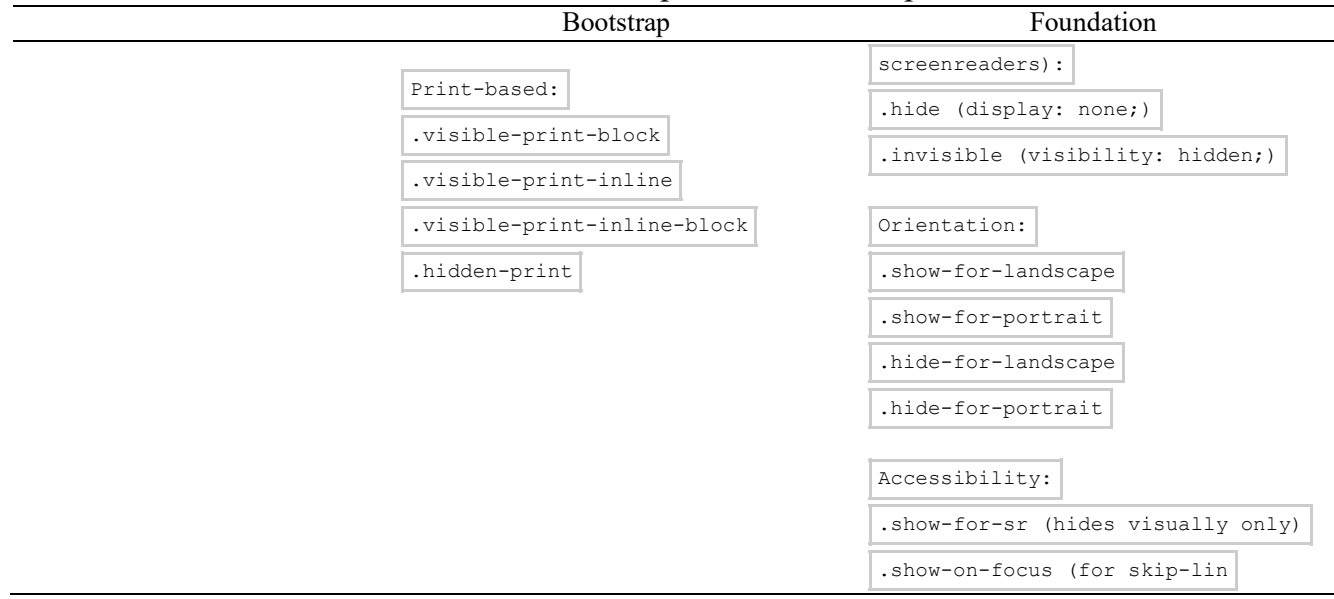

Table 4. User Interface (UI) and Widgets

\begin{tabular}{ccc}
\hline & Bootstrap & Foundation \\
\hline Alerts & Yes & Yes - Callout \\
Breadcrumbs & Yes & Yes \\
Buttons & Yes & Yes \\
Carousel & Yes & Yes - Orbit is back \\
Collapse/Accordion & Yes - Collapse & Yes - Accordion \\
Dropdown & Yes & Yes - Dropdown, Drilldown, \& Accordion menus \\
Forms & Yes & Yes \\
Form Validation & Yes & Yes - Abide \\
Grids & Yes & Yes \\
Icons & Yes & Yes - with download \\
Labels & Yes & Yes \\
Pagination & Yes & Yes \\
Panels & Yes & now Cards \\
Progress bars & Yes & Yes - Callout \\
Responsive media & No & Yes \\
Tables & Yes & Yes - Interchange \\
Tooltips & Yes & Yes \\
\hline
\end{tabular}

\section{DISCUSSION OF RESULTS}

In this section we depict the differences between Bootstrap and Foundation. We presents trough Table 5 the main results derived from our study.

Table 5. Results of Comparison

\begin{tabular}{|c|c|}
\hline CSS Preprocessor & $\begin{array}{l}\text { Both Foundation } 6 \text { and Bootstrap } 4 \text { frameworks are based on SASS. They both have a good set of } \\
\text { mixins, a separate parameter sheet, reusable components, and so on. }\end{array}$ \\
\hline JavaScript & $\begin{array}{l}\text { The Bootstrap } 4 \text { and Foundation } 6 \text { JavaScript libraries are written on ES6, which means that the } \\
\text { devellopper does not need Babel (or any other transpiler) if it wants to individually include each } \\
\text { of their JS tools in its development pipeline. However, they both have ES5 transpired versions of } \\
\text { each component. }\end{array}$ \\
\hline Stability & $\begin{array}{l}\text { The Bootsrap team takes stability and backward compatibility seriously. They use Saucelabs' } \\
\text { automated testing tools to ensure that everything passes a test before engaging in their main } \\
\text { branch. The result is a set of very stable tools that can be used as a daily driver. } \\
\text { Foundation, on the other hand, was released as "stable" a little over a year ago. At first launch, } \\
\text { some components were unfinished, like Abide (their form validation library) which was } \\
\text { completely broken, so their color palette settings. From } 6 \text { to } 6.3 \text {, the team changed a number of } \\
\text { things by making some settings obsolete and introducing things like ES6 to } 6.2 \text {. The offcanvas } \\
\text { has been reorganized and the parameters are very flexible in } 6.3 \text {. } \\
\text { Since Foundation } 6 \text { has been released, its stability is now excellent. The components seem to be } \\
\text { well tested and the team publishes updates almost every } 3-4 \text { months. }\end{array}$ \\
\hline
\end{tabular}


Table 5. Results of Comparison (continue)

\begin{tabular}{|c|c|}
\hline CSS Preprocessor & $\begin{array}{c}\text { Both Foundation } 6 \text { and Bootstrap } 4 \text { frameworks are based on SASS. They both have a good set of } \\
\text { mixins, a separate parameter sheet, reusable components, and so on. }\end{array}$ \\
\hline Grid & $\begin{array}{l}\text { Bootstrap and Foundation support responsive grids and flexible breakpoints. However, } \\
\text { Foundation supports responsive gutters, which are so easy to configure and seem magical. } \\
\text { Collapsed gutters are also a thing in Foundation. The developer may have or remove gutters in } \\
\text { specific cases that do not require them simply by adding a class. Other things like centered } \\
\text { columns and block grids make the Foundation the winner in grids. } \\
\text { As far as backward compatibility is concerned, Foundation and Bootstrap use the same class } \\
\text { convention as their previous versions. This makes the migration as painless as possible. } \\
\text { Flexbox grids are also available on Foundation and Bootstrap as a separate setting that the } \\
\text { developer can turn on / off based on support for their target browser. }\end{array}$ \\
\hline forms & $\begin{array}{l}\text { In Bootstrap } 4 \text {, there are special classes for radios and checkboxes that will make them much } \\
\text { better than the default ones of the browser. Online forms, validation icons, and icon labels are also } \\
\text { very powerful on Bootstrap. } \\
\text { Foundation } 6 \text { has a simpler form layout that is heavily dependent on the grid. Online forms do not } \\
\text { exist in the Foundation, which is almost missed. In addition, predefined fields are prohibited on } \\
\text { Foundation, so the developer must rely on external CSS to justify its selection boxes, radio } \\
\text { buttons and checkboxes. }\end{array}$ \\
\hline Menus & $\begin{array}{l}\text { While Bootstrap } 4 \text { has the same old drop-down lists, tabs, and basic navigation menus that we } \\
\text { learned from version } 3 \text {, Foundation has dramatically improved their drop-down lists by including } \\
\text { other variations that might be useful in some case. }\end{array}$ \\
\hline Browser support & $\begin{array}{l}\text { Both frameworks support the following browsers: Chrome (Mac, Windows, iOS and Android), } \\
\text { Safari (Mac and iOS only), Firefox (Mac and Windows) and Opera (Mac and Windows). } \\
\text { However, there is a slight difference with regard to Internet Explorer - Bootstrap supports IE8 and } \\
\text { newer versions, while Foundation starts at IE9 plus newer versions. }\end{array}$ \\
\hline CSS preprocessors & $\begin{array}{l}\text { Bootstrap includes SASS and LESS files, which means that it can be used in applications that use } \\
\text { one of these CSS preprocessors. On the other hand, Foundation only supports SASS. }\end{array}$ \\
\hline
\end{tabular}

After the comparative study of the two front-end frameworks Bootstrap and foundation, we see that each framework has its own features and benefits and the choice between these two frameworks depends on the needs of the developer's web application.

To help the developer choose the right framework for the design of his project, we present the main advantages of each framework: the first one is Bootstrap:

a. Massive community support: Given the huge popularity of Bootstrap, there is a large community using the Framework. Therefore, if the developer has a question about Bootstrap, he is more likely to find quickly an answer. Further, there are more tutorials as well as full courses on how to use Bootstrap.

b. Other themes available: Because of its popularity, there are other Bootstrap themes. This means that if the developer uses a platform like WordPress and is looking for a theme to use, this might be a better option.

c. Better browser compatibility

d. Can use Less or Sass

Many professional websites have used Bootstrap to create elegant and functional pages. Among them: Newsweek, Vogue and Lyft. We present trough Figure 6, the statistics about the use of this framework.

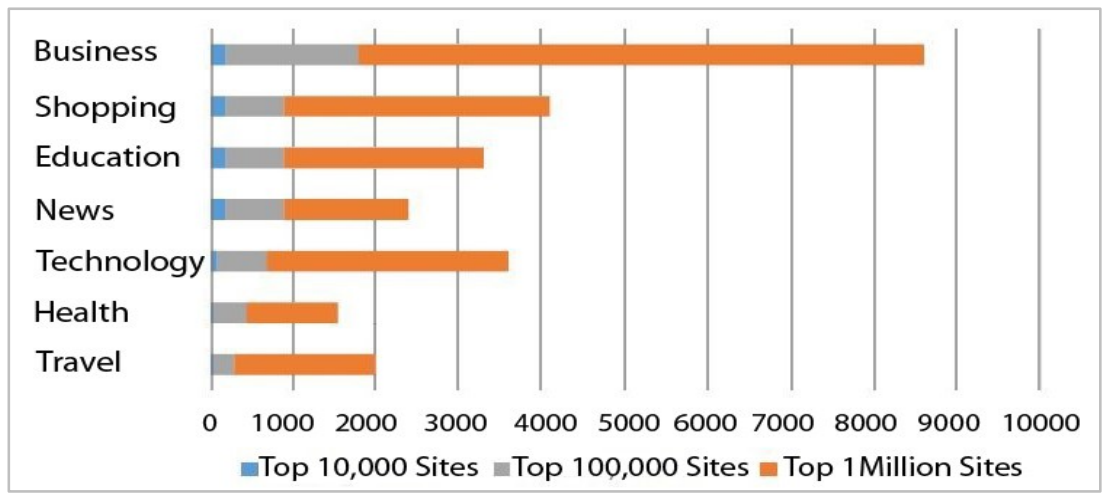

Figure 6. Bootstrap popular website 
Likewise, we present the advantages of Foundation Framework:

a. More than one design approach by the developer: Foundation provides a better environment for customizing the web site. Although it is possible to customize with Bootstrap, it takes a lot of effort for the developer site to look like other Bootstrap sites. With Foundation, the detached CSS design is a lightweight design and the developer does not have to put a lot of effort into customizing it.

b. Foundation does not need to add classes to be responsive or have a certain style: If the developer does not add the correct CSS classes with Bootstrap, the effects do not appear. With Foundation, there are built-in basic CSS appearances. Some may prefer this, especially since there will not be as much CSS in its HTML.

c. More features of the grid system: Many prefer the Foundation grid system because they find it offers more flexibility. Some additional abilities :

1. The ability to center columns

2. The developer can add a "collapse" class that allows him to easily edit columns and remove gutters.

3. Can use a block grid that allows you to create columns of equal size with minimal markup.

d. Other built-in widgets: With regard to additional components and JS extensions, Bootstrap and Foundation are basically equal. Here are some extra that Foundation.

1. Form validation, Abide : HTML5 form validation library

2. Menus off canvas : the developer easily places the navigation of his site on the side of his site

3. Pricing tables : ready-to-use table, designed to display the prices of a product by subscription Responsive media

4. Reactive media, exchange : the website loads different media for different devices / screen sizes, so that the developer can customize the user experience

5. Support from right to left : the developer the direction of the text ; Ideal for sites in other languages where characters move from right to left

6. Sites using Foundation as shown in Figure 7: Just like Bootstrap, Foundation is used by big names. Among them: PBS, National Geographic, The Washington and PostMozilla.

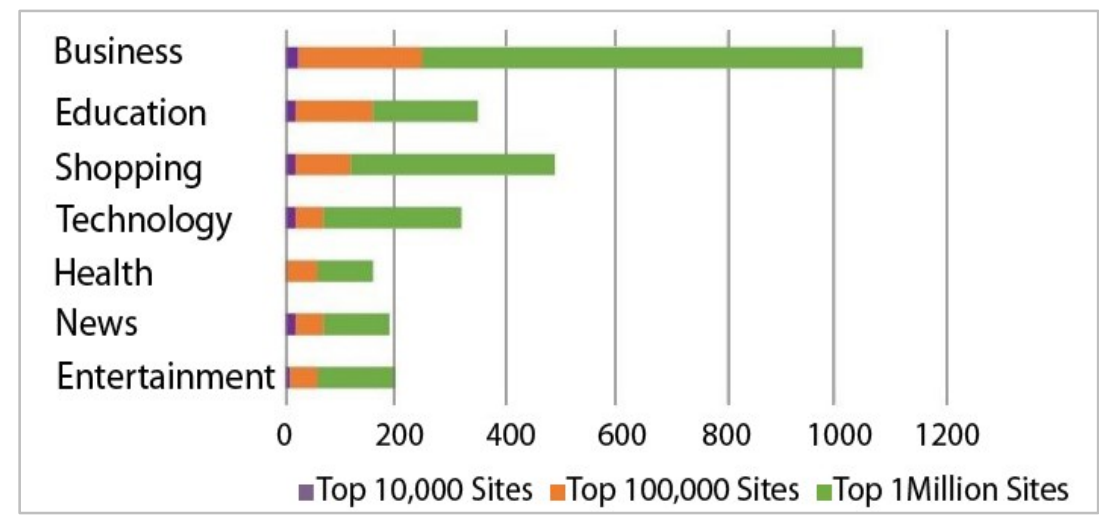

Figure 7. Foundation popular website

\section{CONCLUSION}

Using a front-end framework is great for people who want to create content quickly, or at least not doing it from scratch. Without a doubt, the use of a front-end framework facilitates the work of the developer. Many web sites use Bootstrap and Foundation because they can both meet the needs of developers, and to choose the right framework. In this context this paper provided a detailed analysis of these two frameworks, to enhance the process of frameworks selection. The results of this contribution provide guidelines to front-end developers to select the appropriate framework based on the framework popularity, the active development of the framework, framework maturity, framework documentation, and finally the level of specificity of the framework. 


\section{REFERENCES}

[1] D. R. Lakshmi and S. S. Mallika, "A Review on Web Application Testing and its Current Research Directions," International Journal of Electrical and Computer Engineering (IJECE), vol. 7, no. 4, p. 2132, 2017.

[2] W. D. Cletus, A. Kakandar, and C. N. Paul, "Responsive Web Design Frameworks : A Review," Int. J. Sci. Res. Comput. Sci. Eng. Inf. Technol., vol. 4, no. 5, pp. 132-136, 2017.

[3] W. Computer, "Responsive Web Design With Bootstrap," vol. 5, no. 4, p. 274, 2016.

[4] N. Jain, "Review of Different Responsive CSS Front-End Frameworks," J. Glob. Res. Comput. Sci., vol. 5, no. 11, pp. 5-10, 2014.

[5] "Introduction aux frameworks front-end (Bootstrap, Foundation) - alticreation." [Online]. Available: https://www.alticreation.com/bootstrap-foundation-frameworks-front-end/.

[6] S. Subramaniam, S.-C. Haw, and P. K. Hoong, "Bridging XML and Relational Databases: An Effective Mapping Scheme based on Persistent Labeling," International Journal of Electrical and Computer Engineering (IJECE), vol. 2, no. 2, pp. 239-246, 2011.

[7] S. K. Patel, "Responsive Web Design with AngularJS," p. 142, 2014.

[8] N. Edan, A. Al-sherbaz, and S. Turner, "Design and Implement a Hybrid WebRTC Signalling Mechanism for Unidirectional \& Bi-directional Video Conferencing," vol. 8, no. 1, pp. 390-399, 2018.

[9] "Les meilleurs frameworks CSS pour créer un site web professionnel. | Outils-web.fr." [Online]. Available: http://outils-web.fr/les-meilleurs-frameworks-css-pour-creer-un-site-web-professionnel/.

[10] "COMPARAISON ENTRE LES FRAMEWORK FRONT-END BOOTSTRAP ET FONDATION | SUPINFO, École Supérieure d'Informatique." [Online]. Available: https://www.supinfo.com/articles/single/4903-comparaisonframework-front-end-bootstrap-fondation.

[11] “Tutoriel Bootstrap: les premiers pas - 1\&amp;1." [Online]. Available: https://www.1 and1.fr/digitalguide/sitesinternet/developpement-web/tutoriel-bootstrap-les-premiers-pas/.

[12] "Responsive CSS Framework Comparison | Vermilion Design + Digital." [Online]. Available: https://www.vermilion.com/responsive-comparison/. 